satisfaction of elucidating the structures of these beautiful molecules, such compounds have provided a range of interesting theoretical valency problems and have stretched to the limits the techniques currently available to chemists for characterizing molecules. In addition, they have stimulated the interest of industrial chemists and molecular biologists: the former because of the potential of the compounds as homogeneous and heterogeneous catalysts, and the latter because iron sulphur clusters have been found to be important components of ferredoxin and nitrogenase.

Transition Metal Clusters therefore represents a timely attempt to bring together under one cover some of the developments in this area.

The book covers most of the important aspects of cluster compounds, with P.R. Raithby reviewing the geometries of more than 1,000 cluster compounds whose structures have been elucidated using $\mathrm{X}$-ray and neutron diffraction techniques. K. Wade and R.G. Wooley have contributed two very different views of the bonding in these polyhedral molecules, J.A. Connor has provided a balanced and critical account of the thermochemical estimation of metal-metal bond strengths in cluster compounds, and B.F.G. Johnson and R.E. Benfield have discussed their fluxional characteristics. The bio-

\title{
Animals: their use and their abuse
}

\section{Michael F.W. Festing}

Animal Suffering: The Science of Animal Welfare. By Marian Stamp Dawkins. Pp.149. Hbk ISBN 0-412-22580-8; pbk ISBN 0-412-22590-5. (Chapman \& Hall: 1980.) Hbk £7.50; pbk £3.95.

ON AVERAGE, a person living in a developed country such as the UK will be responsible for the death of about 600 chickens, 22 pigs, 20 sheep and 7 cows for food and 5 mice, 2 rats, 1 chicken, a third of a guinea-pig and two-hundredths of a cat and dog for medical research during their 75-year life span. They will also be responsible for the deaths of countless fish, both for food and for sport. Their food supplies and health will also be maintained at the cost of the death by poison or trapping of an unknown, but large number of mammals and birds classified as pests. Who can doubt that the production, maintenance and killing of all these animals involves a certain amount of suffering? The problem for us as individuals, and as a society, is to decide what to do about it, if anything.

In order to make some progress, we must sort out our moral attitudes to animals and to our fellow human beings, and we must

logical significance of cluster compounds is stressed by C.D. Garner, in a chapter dealing with cubane clusters, and $\mathbf{R}$. Whyman has attempted to evaluate whether clusteı compounds do actually function as effective catalysts by reviewing the relevant academic and patent literature. There is also a detailed review by A.J. Deeming on the reactions of metal carbonyl cluster compounds.

The book gains by drawing on the expertise of the authors to provide a reasonably up-to-date (literature references up to the end of 1978) and broad view of the field, but suffers from an uneven view of the potential readership of the book. Thus although some chapters are written at a level which would be appreciated by a third-year undergraduate, others are in the style of a research paper or a terse contribution to a Chemical Society Specialist Periodical Report. Therefore, although I wholeheartedly agree with the editor that this book will serve as a "source of reference to workers in the field", I very much doubt if it will be used much as "an advanced text for undergraduates or graduates in chemistry".

Michael Mingos is a Lecturer in Inorganic Chemistry and Fellow of Keble College at the University of Oxford. He was recently awarded the Corday Morgan Medal of the Chemical Society for his experimental and theoretical contributions to organometallic and cluster chemistry. into a battery cage, but this does not reduce the suffering of those already in occupation. In fact, reading these chapters it becomes clear that there is no single way in which the suffering of an animal can be clearly quantified. Ideally, it would be nice to be able to measure some physiological or biochemical variable which could be used as an index of "stress". Unfortunately, although certain parameters can be measured which give an indication of the state of the general adaptation syndrome, there is no evidence that a complete absence of "stress" (which can itself be defined in many different ways) is necessarily beneficial to an animal. Nor can we say that an animal suffers because it is forced to lead an "unnatural" life. In nature many animals have a relatively short life-span, quickly succumbing to predators, disease, exposure or starvation. Their death may be lingering and painful. Even allowing animals to choose the conditions under which they will live does not always help. Animals sometimes choose a course of action which is not in their best interests; few domestic pets enjoy a visit to the vet, even though this is usually for their benefit.

Does this mean that we are unable to assess animal suffering at all? Dr Dawkins is clearly concerned that the general tone of these early chapters "may have seemed rather negative", and she goes on to provide a list of questions which we must ask ourselves in order to determine whether an animal is suffering unduly. We must consider the conditions in which the animals are kept, whether they are healthy, whether they appear physiologically or behaviourally abnormal, and if so what is likely to be the cause of these abnormalities. If possible we should also determine what conditions the animals themselves prefer. And in the end, the assessment will have to be subjective. "There is no formula for giving a clear yes/no answer to the question of whether an animal is suffering". Even if we could answer the question unequivocally, we would still have to decide how much suffering we should permit in relation to the benefits we obtain from animals. That, however, is a whole new subject which falls more in the field of ethics than biology.

Dr Dawkins has produced an excellent review of the state of our ignorance about suffering in animals, and has highlighted many inconsistencies in our attitudes. This book should be read by all those involved with farm and laboratory animals, in the hope that they will endeavour to reduce needless suffering wherever it occurs. If we must cause suffering to animals in the name of agriculture or medical research, let us at least make sure that it is not caused unnecessarily through our ignorance of the needs of those creatures that provide us with so many benefits. agriculture, is more concerned with economics than animal welfare. A higher productivity (i.e. profit) may well be achieved by putting an additional chicken 